\title{
ANALYSIS OF PATENT \#US2014/0319274A1: A CASE STUDY OF SIMULATIONS FOR NEW DESIGNS REVIEW
}

\author{
Roca-Gonzalez, J. L.; Vera-Lopez, J. A. \& Rodriguez-Bermudez, G. \\ University Centre of Defence at Spanish Air Force Academy, \\ C/ Coronel López Peña, 30720 Santiago de la Ribera (Murcia), Spain \\ E-Mail: jluis.roca@cud.upct.es, juanantonio.vera@cud.upct.es, german.rodriguez@cud.upct.es
}

\begin{abstract}
The technical information needed to replicate a prototype is usually concealed when aircraft manufacturers patent new designs. Competitors must face this problem by developing inverse engineering processes that are validated by simulations and design thinking processes to find any patent weaknesses.

This paper focuses on a case study for patent analysis, applied to a revolutionary new concept in aircraft design: Patent \#US2014/0319274A1 "Aircraft including a passenger cabin extending around a space defined outside the cabin and inside the aircraft". The computer simulations were performed first by a low computational software, "FlowDesign" (Autodesk), and afterwards, by a professional tool "3DWind tunnel" (MicroCFD). Results revealed that the drag coefficient and the drag force decreased $5.8 \%$ and $3.8 \%$ respectively at climbing, while the lift force increased up to $47 \%$ for a pitch angle of $6^{\circ}$. The patent weaknesses reside in a wrong seat arrangement and not taking into account the possibility of a "hole" variable to adapt aircraft shape to different flying operations.

(Received in November 2017, accepted in May 2018. This paper was with the authors 2 months for 2 revisions.)
\end{abstract}

Key Words: Patent Analysis, Aircraft Design, Improvement Characterization

\section{INTRODUCTION}

The incoming aeronautical framework for the future aircraft design requirements is forcing the most relevant manufacturing companies to develop an active research policy on innovation in order to reach leading positions over competitors. These innovations are protected from being copied thanks to the legal coverage of patenting.

Innovation, as a concept, is commonly based on ideation, which is defined as a complex activity at the core of design thinking where the technical information between designers is intertwined with draft and design tools [1]. This results most of the times in patents that may seem too futuristic and far from current usability. In spite of this, the legal coverage of patents ensures technological leadership when new circumstances appear (e.g. new material developments, new social trends, etc.) that allow these patents to become feasible inventions. Back to aeronautical manufacturing, this point of view has been the main argument used by big companies, such as Airbus or Boeing, to publish the results of innovation through ideation; therefore, several patents are signed every year even if there is no commercialization purpose planned in the near future.

Design research, when abridging ideas of innovation in patent reports, requires a high creative skill to transform drafts and outlines into professional reports that should include enough technical information to summarize the main desired advantages of the patent. For this reason, patents are written after creating a design through problem solving processes that remark the relevance of understanding the design principles. This statement has been used by some researchers to warn educational background managers about the risk of future generations of engineers that could be unprepared to create useful designs if they have not been thoroughly trained in this matter [2]. On the one hand, legal patents under design analysis offer an excellent source of case studies to provide examples that may encourage other researchers, or university educators, to carry out studies in design thinking applied to 
design engineering [3]. On the other hand, the patents' legal coverage to protect ideations may be a contradiction in itself, since its publication reveals future companies' design proposals offering strategic information that may lead competitors to develop countermeasures to these future innovations.

This paper is an extended case study on patent analysis with two main scopes: the first scope is to offer an example of how the process of technical reviewing, applied to patent analysis, could be carried out by a competitor to acquire a better understanding of a new idea, its advantages and its possible design weaknesses; and the second scope focuses on the relevance of inverse engineering, which takes advantage of design, modelling and computer simulation to transform a limited information input (simple drafts extracted from a pdf file) into technical data. Regarding computer simulations, it is necessary, first of all, to state clearly that a limited source of information affects results in such a way that they may not be validated by its absolute value. However, the strategy of using simulation results to contrast model variances allows validating results when expressed as a percentage of an increasing or decreasing value of one model in relation to another selected as a baseline [4]. Appling this to the case study, two models were drawn, one with a hole in its centre, and another one without it, with the main purpose of referring results of the model with a hole to the one without it.

This paper is structured as follows: a first section is focused in analysing the information regarding patent \#US2014/0319274A1 "aircraft including a passenger cabin extending around a space defined outside the cabin and inside the aircraft"; the second section is dedicated to set and perform flow computer simulations to extract technical information, the results of which are later discussed in the conclusions section, in order to provide an understanding that ends with a design improvement proposal.

\section{PATENT ANALYSIS}

\subsection{Design thinking applied to design research}

The term design thinking is being used at present to refer to the human-centred open problem solving processes that decision makers use in order to solve real "wicked" problems [5]. In patent analysis, design thinking is a matter of interest for engineering educators [6] since it may be considered as an important tool for novice engineers to develop creativity skills.

The combination between design research and design improvement by patent analysis may be easily achieved by understanding how design requirements could be conciliated to optimize an innovation [7]. Under this scope, design thinking processes have been applied along this paper's structure, firstly by analysing the patent in order to extract as much information as possible, and, later, by applying inverse engineering to draw a real size 3D model plus an alternative design to contrast the simulation results (in this case: a model with a hole in its centre and one without it). The computer simulations were performed with "Autodesk Flow Design", which offers a low computational cost, and afterwards with "MicroCFD 3D Wind Tunnel", professional and advanced simulation software.

\subsection{Source information analysis: Patent \#US 2014/0319274A1}

The information reported when professional reviewing is applied to a patent may be worth an entry in an analysis logbook, which defines not just the underlying structure of data support but also the best practices that focus in design aspects. Therefore, the process of learning by analysing helps students and novice engineers [8] to understand main engineering design principles where minimum information is expected to be enough to replicate models.

The case study selected, "aircraft including a passenger cabin extending around a space defined outside the cabin and inside the aircraft", can be summarized regarding the design 
concept as a complex flying wing with a hole in its centre, the main advantage of which resides in the pressure resistance being increased by decreasing the structural bottom requirements, which, at the end, increases the available space for passengers. Nevertheless, in terms of innovation, flying wings have been deeply analysed in terms of aerodynamics and flying principles [9], so there are some previous contrasted assumptions regarding their advantages which are: lower wetted area to volume ratio, lower interference drag and better aerodynamic results compared to conventional aircrafts. However, the "hole" concept is an innovative design aspect that opens a new review opportunity to test the idea of applying an inverse engineering process on the information that is poorly described in the patent report.

The report shows almost sixteen figures that represent several design views or cross section areas that do not belong to a singular design. Perhaps, it can be deduced that the main purpose could have been to include, under the patent coverage, several manufacturing design options. The patent describes almost six embodiments of the invention (see Fig.1), where figures labelled inside the patent from 0 to 8 (same as in Fig.1, to remain consistent with patent figure titles) represent the first idea of the concept, showing several views of the same flying wing. Figs. 9 to 10 correspond to a second design inside the patent, where the aircraft has been modified to a bigger scale with more than one open structure in its centre. The third embodiment, Fig. 11, shows a difference in the structure delimiting the passenger cabin, without an aft projection. Figs. 12 and 13 are partial top views of a possible fourth and fifth embodiment and Figs. 14 and 15 are cross-sectional views of Fig. 13 while, finally, Fig. 16 is a cross sectional view of a possible sixth embodiment.

\subsection{The inverse engineering process}

The academic scope when analysing different kinds of innovation projects is usually based on teaching basic methodologies in order to customize and optimize product design and development processes [10], which constitutes the academic competences of design engineering at present. However, sometimes when these processes are performed by novice engineers some other competences may be missing such as those acquired after working for a long period of time on multidisciplinary teams.

The analysis of several case studies helps consolidating academic and professional competences [11] when novice engineers or engineering students are required to proceed first with the analysis of a predefined set of performance metrics, assessment methodologies and acceptance criteria in order to obtain an optimal design.

The theory of axiomatic design defines optimal design as the proposal that has the minimum information content while satisfying all the functional requirements [12]. However, in order to optimize a previous design, a full understanding of the original design is required, which includes finding out what information may be missing or concealed, such as the reasons that could have led first designers to choose some options over others. It is under these circumstances that inverse engineering processes help restore the designs to a previous state, in order to develop form-finding procedures which can be supported by design analysis and simulations [13], thanks to computer-aided design systems [14].

In a similar way, inverse engineering is also defined as the common process of revising a set of drawings and reports, submitted (by a contractor when it is applied to an "as built" report) upon completion of the work [15]. Applying this definition to design developments, the inverse engineering process could be defined then as the process of analysing and studying all the information reported that allows recovering a functional model of the design.

The information reported inside patents usually does not provide any technical aspects or definitions, or data tables or any other parameters that could help validate the advantages of the design described in the patent purpose statement. Therefore, it is necessary to perform an inverse engineering study to build a 3D model in order to calculate parameters through 
computer simulation, to identify design advantages based on simulation results rather than descriptions.

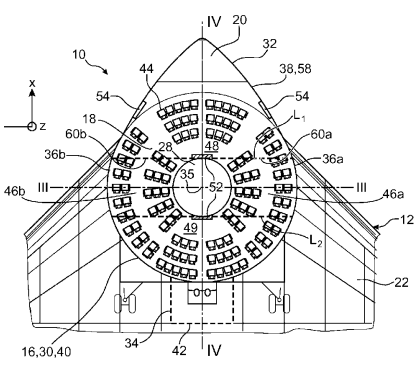

Patent Draw \#0

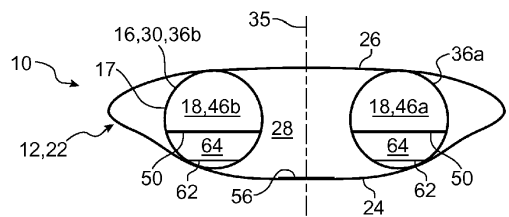

Patent Draw \#3

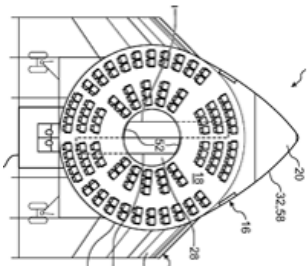

Patent Draw \#6

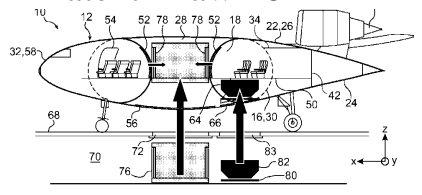

Patent Draw \#7

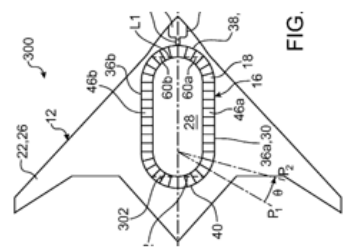

Patent Draw \#12

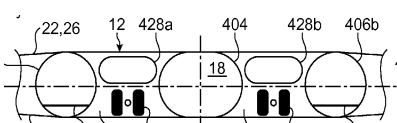

Patent Draw \#14

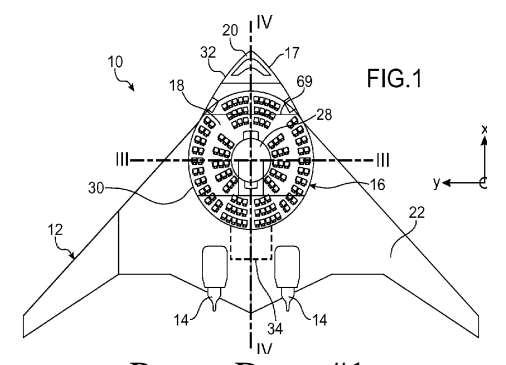

Patent Draw \#1

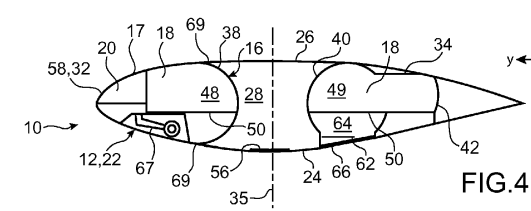

Patent Draw \#4

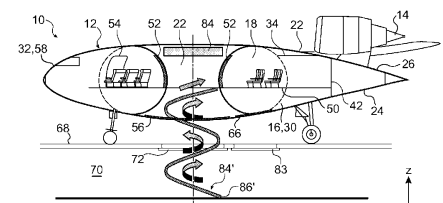

Patent Draw \#8

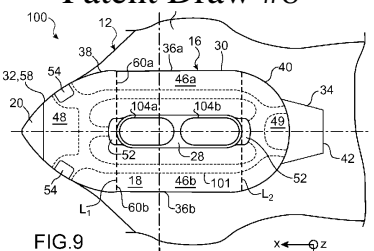

Patent Draw \#9

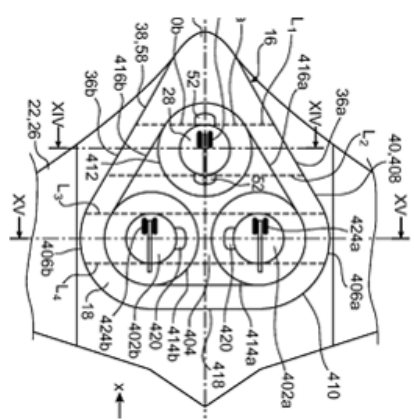

Patent Draw \#13

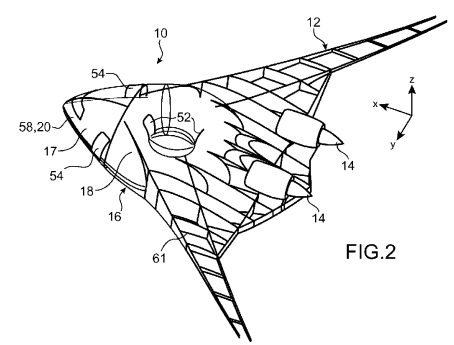

Patent Draw \#2

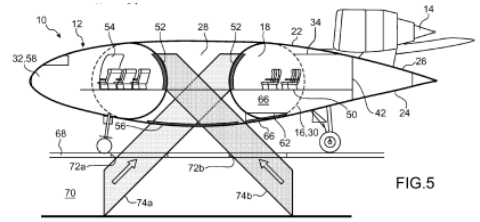

Patent Draw \#5

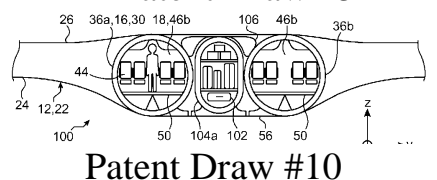

Patent Draw \#10

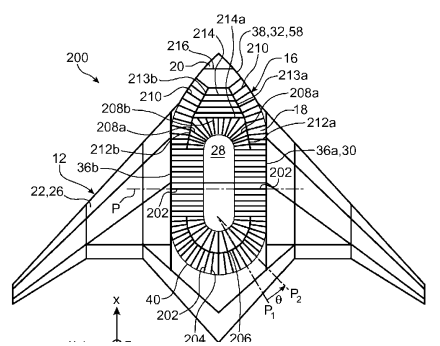

Patent Draw \#11
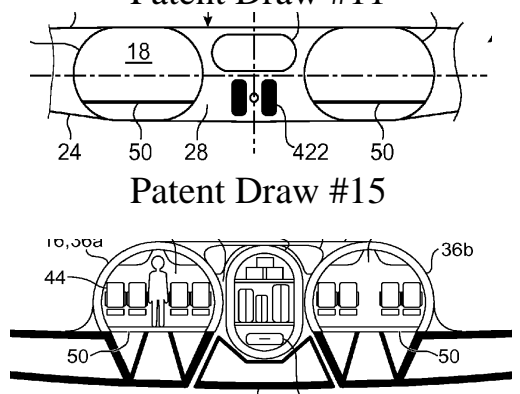

Patent Draw \#16

Figure 1: Representative draws at patent \#US2014/0319274A1. Source: Public information service https://www.google.com/patents/US20140319274.

The reverse process starts with the analysis of the drawings inside the patent, by inserting all the images in a CAD software to build a 3D design, using a step by step methodology which requires a search for external sources of information to extract any data that could be considered in order to scale the model to a real size. This process was conducted by paying special attention to some details inside each drawing, like seating details, suitcases, doors and tires, the size of which has a correspondence in dimension with real objects and so it may help determine a scale factor.

In spite of possible resizing errors that could affect the results of the computer simulation when calculating airflow parameters, this research focused on the idea of comparing designs (one with the functional hole and other without it) in order to establish that any scale errors or 
any lack of precision while drawing the 3D model will affect both models in the same way, which means that the simulation results could be accepted if the results between designs are cross referenced.

Finally, in order to increase the model accuracy as much as possible, it was assumed that the seats in the drawings followed the last trend in sizing dimension of a relevant aircraft manufacturer. Thanks to technical papers such as "airplane characteristics for airport planning" of Airbus, it was established that the actual seat size of the design corresponds with the latest Airbus trend, where the seat places are enlarged for economy class, from 17 inches to 18 inches (measured from the middle of one arm rest to the other). The final sizing of the patent design (see Fig. 2) created a model with $88.58 \mathrm{ft}(27 \mathrm{~m})$ of length, $150.92 \mathrm{ft}(46 \mathrm{~m})$ of wingspan, $22.97 \mathrm{ft}(7 \mathrm{~m})$ of maximum fuselage height, $12.5 \mathrm{ft}(3.81 \mathrm{~m})$ of cabin height and $19.69 \mathrm{ft}(6 \mathrm{~m})$ of cabin width. The full model was drawn with AutoCAD 2013 by attaching figures of the pdf file as a background layer so poly-lines could be easily drawn to follow the pdf edges and, therefore, to make a wire structure to be transformed into a 3D model by solid making tools, "loft", "extrude", "union", "intersect" and "subtract" among other commands.

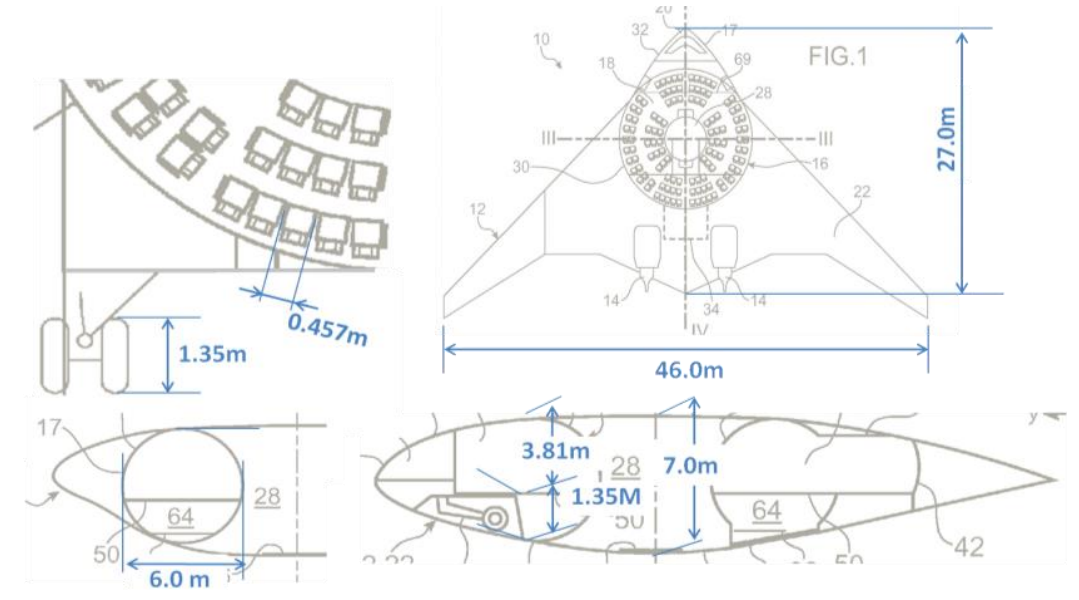

Figure 2: Useful dimensions extracted from the patent designs. Source: \#US2014/0319274A1 patent.

\section{COMPUTER FLOW SIMULATIONS}

\subsection{Flow simulation by Autodesk}

Autodesk provides free software with high technical tools for engineering students. Some of these tools may have computing limitations but can be downloaded just by signing in as a university member or a member of any other higher education centre. Among all the Autodesk utilities of high interest for designers, there is a wind tunnel simulator, the "Flow Design (FD)" (its latest version \#103.2014.04.29), that calculates the drag coefficient and drag force by using a transient flow solver for subsonic flows, it shows transient (timedependent) results that might not otherwise be seen by the user in a steady-state simulation.

The drag coefficient is a non-dimensional parameter that depends on the Reynolds number and represents the drag results given by an object's geometry and its form and its skin friction drag, so lower values means less aerodynamic resistance to air flow and therefore a best design feature. The main disadvantage of the Autodesk Flow Design software is that the simulation is limited to subsonic flow $(<150 \mathrm{~m} / \mathrm{s})$ and it is not able to set up any other boundary conditions beyond the simulation resolution and the wind velocity. Therefore, the scientific accuracy of the results could be discussed when contrasted against other results extracted from real wind tunnel experiments.

However, when this software is used to evaluate similar models (same boundary conditions), it may be an easy tool to assess whether design changes can be considered as an 
improvement or a worsening in relation to an original design just by measuring the differences on drag coefficient for both models (the original and the proposed one).

The drag coefficient can be plotted against the pitch angle by running several simulations with different pitch angles and velocities thanks to a complicate data post processing, where quadratic regressions (see Fig. 3) may be easily estimated. In the case study, these equations were labelled as $f(\propto)$ for results corresponding to the 3D model without a hole in its centre and $\hat{f}(\propto)$ for the model with a hole, so both equations could be compared and analysed afterwards.

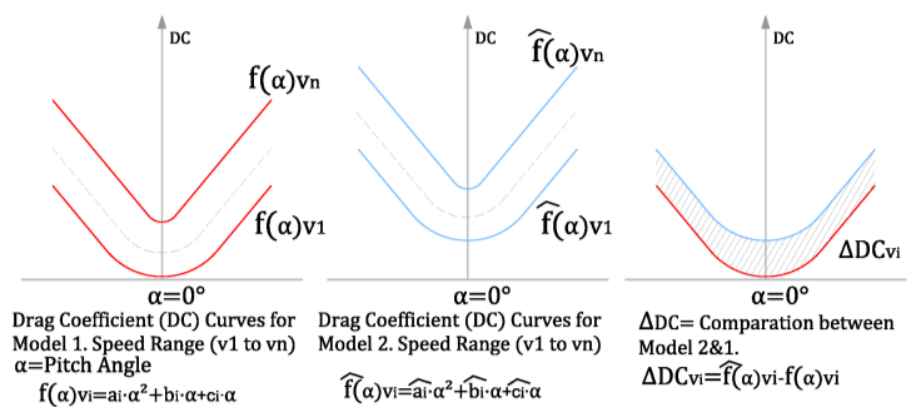

Figure 3: Examples of drag coefficients plot vs pitch angle for both models.

\subsection{Flow simulation by MicroCFD}

The MicroCFD Virtual Wind Tunnel software suite analyses 2D, axis-symmetric, and 3D aerodynamic flow over a wide range of Mach numbers from low subsonic (Mach 0.1) to hypersonic (Mach 10) speed. It runs a boundary-layered modelled, viscous, compressible flow solver for high Reynolds numbers, unsteady flow with fully turbulent wakes. This software has been validated through experimental data in several studies published on the MicroCFD website (www.Microcfd.com) and has been put to the test for over a decade by a diverse number of clients in academia, government, and industry.

The 3D Virtual Wind Tunnel (VWT) used in this design study imports stereo lithography (STL) files, a standard cad format based on surface triangulation. Once an ASCII or binary STL file has been opened with the VWT, it can be visually inspected and checked for validity. Common problems with STL output from cad software include improper or inconsistent orientation of triangular facets, triangles that have collapsed onto lines, and facets with missing, inverted, or otherwise incorrectly oriented outward normal vectors. The MicroCFD VWT is fairly robust in regard to minor gaps in triangular surface definition, unlike other commercially available CFD software packages.

Once the STL file is loaded into the VWT, the model can be flipped and rotated around any axis until it is properly pointed in the direction of the wind, undergoes surface refinement and flow field meshing, and has its flow properties set with just a few mouse-clicks. The user then has the choice to run the simulation on either single or multi-core CPU, or on an NVIDIA compatible graphic processor unit with hundreds of CUDA-cores. Typical run time on a high-end PC is four to six hours. The simulation stop time is automatically pre-set based on the setup conditions and yields 95 percent or better convergence of aerodynamic forces and moments, which can be monitored in pre-set or custom simulation time step increments.

After the computation is completed, the flow field can be sliced in any plane along the major axes to insert stream particles or show contour plots of local Mach number, pressure, density, temperature, or entropy. Likewise, the model itself can be coloured and this feature is present in any of the available flow properties. The Cartesian mesh consists of 31 million fluid and solid cubic cells, equally divided into near field and far field of the flow. At the fluid-solid interface, the cubic cells are cut according to the refined surface triangles such that proper boundary conditions can be defined. The relative size of the model with respect to the 
near- and far-field of the flow is set by the software in order to always achieve optimum results.

A visualization of near-field flow around the model being investigated is shown in Fig. 8, which is embedded into a far-field five times its cubic size. Although not seen in the figure, the far-field flow can be made visible. The diameter of the stream particles was set to one grid unit and thus represents the size of the cubic fluid cells and determines the fineness of the uniform Cartesian near-field mesh. Unlike many other CFD applications, which generally smooth the visualized results of the computed flow while disguising the underlying mesh, MicroCFD maps all colour contours directly onto the mesh, thus preserving the discrete nature of the solution. This helps to better judge the quality of the computed flow.

\section{FLOW SIMULATION RESULTS}

Results for flow simulations were collected in Table I (Autodesk Flow Design) for drag force coefficients and Table II (MicroCFD Virtual 3D Wind tunnel) for lift force results in relationship several speed and pitch angle options.

Table I: Data collection table for drag force coefficient calculations.

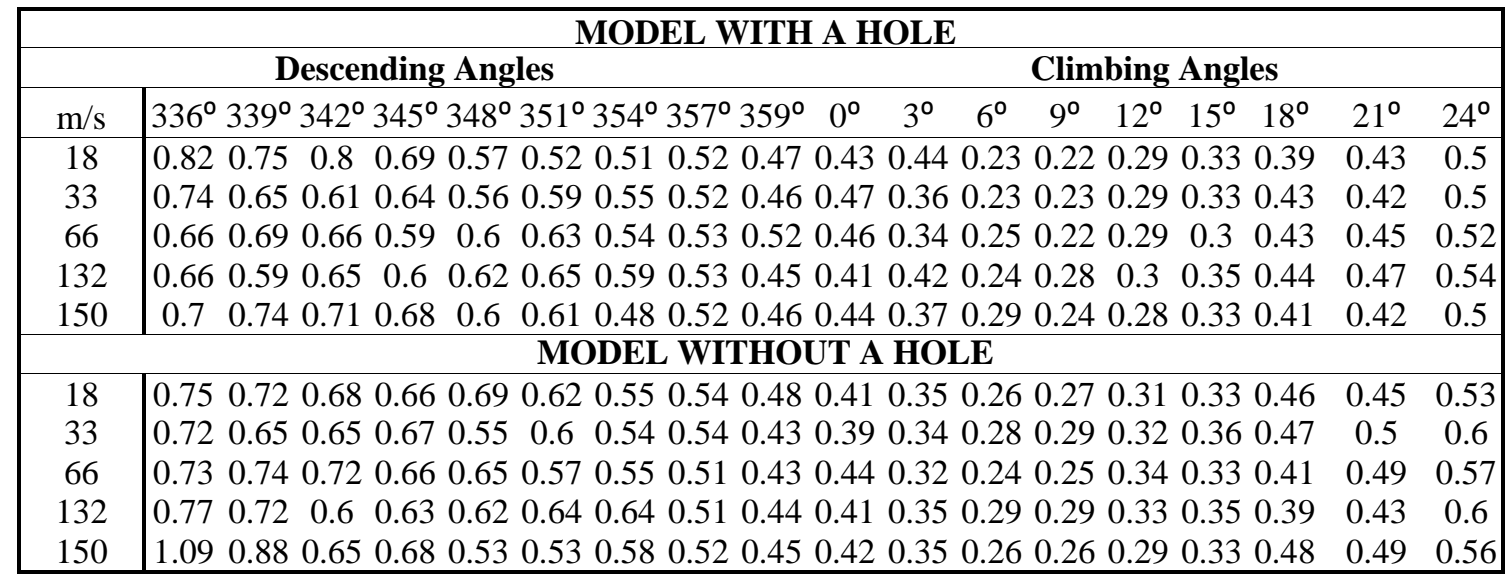

Table II: Data collection table for lift force calculations.

\begin{tabular}{|c|cccc|cccc|}
\cline { 2 - 9 } \multicolumn{1}{c|}{} & \multicolumn{4}{c|}{ MODEL WITHOUT A HOLE } & \multicolumn{4}{c|}{ MODEL WITH A HOLE } \\
\hline Speed & \multicolumn{3}{c|}{ Ascending Pitch Angles } & \multicolumn{4}{c|}{ Ascending Pitch Angles } \\
\cline { 2 - 9 }$($ Mach) & 6 & 10 & 18 & 24 & 6 & 10 & 18 & 24 \\
\hline 0.2 & $-8.91 \mathrm{E}+04$ & $<<0$ & $<<0$ & $<<0$ & $-5.88 \mathrm{E}+04$ & $<<0$ & $<<0$ & $<<0$ \\
0.3 & $-1.24 \mathrm{E}+05$ & $4.70 \mathrm{E}+05$ & $3.21 \mathrm{E}+06$ & $2.34 \mathrm{E}+06$ & $-6.80 \mathrm{E}+04$ & $4.26 \mathrm{E}+05$ & $2.85 \mathrm{E}+06$ & $2.05 \mathrm{E}+06$ \\
0.46 & $-2.215 \mathrm{E}+05$ & $1.19 \mathrm{E}+06$ & $3.92 \mathrm{E}+06$ & $5.75 \mathrm{E}+06$ & $-9.47 \mathrm{E}+04$ & $1.01 \mathrm{E}+06$ & $3.48 \mathrm{E}+06$ & $4.91 \mathrm{E}+06$ \\
0.7 & $-1.04 \mathrm{E}+06$ & $2.34 \mathrm{E}+06$ & $9.29 \mathrm{E}+06$ & $1.34 \mathrm{E}+07$ & $-6.80 \mathrm{E}+05$ & $2.05 \mathrm{E}+06$ & $8.01 \mathrm{E}+06$ & $1.14 \mathrm{E}+07$ \\
\hline
\end{tabular}

\subsection{Flow design results by Autodesk}

When the simulation boundary conditions are unknown, some assumptions need to be made in order to set the software parameters; therefore, it was assumed that the climbing/descending rates for the design were close to standard rates for similar aircrafts, which means that the pitch angles to be studied were between $0-6^{\circ}$ to $24^{\circ}$ for climbing rates and $-1^{\circ}\left(359^{\circ}\right)$ to $-24^{\circ}\left(336^{\circ}\right)$ for descending rates. The five levels of flying speed were studied between $40 \mathrm{mph}(18 \mathrm{~m} / \mathrm{s})$ and the highest speed available for the simulation software which is $336 \mathrm{mph}(150 \mathrm{~m} / \mathrm{s})$.

The wind tunnel size was set as recommended by Autodesk, inside a box two times the aircraft length at the front and four times the same distance at the back. The model must also 
be centred inside the tunnel with a free space on both sides of at least two times the aircraft width and at least two times the maximum aircraft height above and below, which at the end implied a size of $230 \mathrm{~m}$ (width) $\times 189 \mathrm{~m}$ (length) $\times 35 \mathrm{~m}$ (height). A first overview of the results (see Fig. 4) revealed that the model with the hole provides a slight improvement for the drag coefficient but just for some descending and climbing angles.

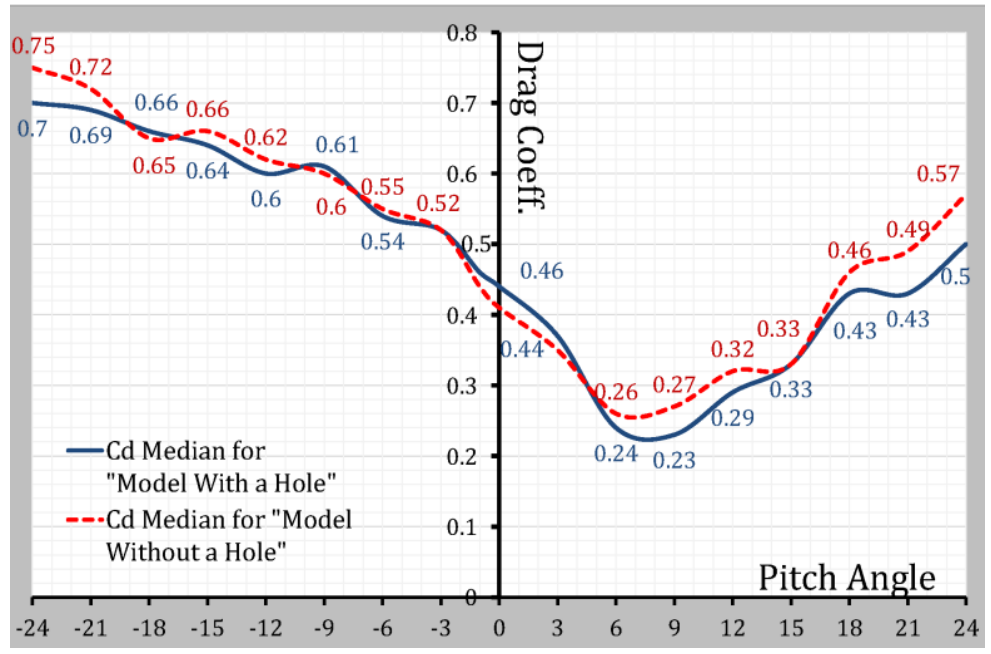

Figure 4: Median values of drag coefficient for a speed range of $18-150 \mathrm{~m} / \mathrm{s}$.

However, for pitch angles between $-3^{\circ}$ and $+6^{\circ}$ the model without the hole produces similar values in a speed range between $18-150 \mathrm{~m} / \mathrm{s}$. This circumstance was taken into account when defining the improvement factor as the difference of drag coefficients for each model comparing with the model without the hole. In order to perform this comparison, the simulation was set for a speed range as shown in Table I, which results were adjusted afterwards by applying quadratic regressions for each case (see Table III). The acceptance criteria was established by the coefficient of determination with a minimum of 0.75 , requiring to make different regressions for climbing and descending pitch angles.

Table III: Quadratic regressions and final results of drag coefficient dependent on pitch angle.

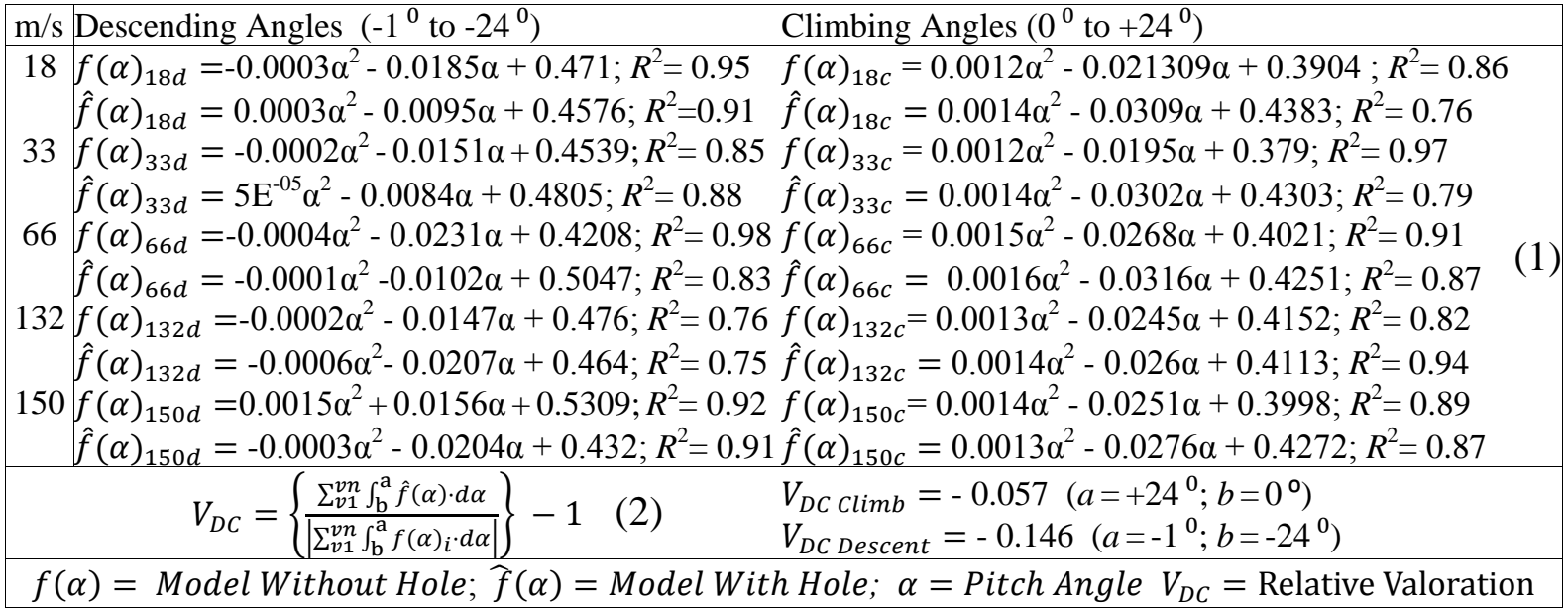

Regarding the drag force, (see Figs. 5 and 6) there are also some pitch angles where the model without the hole provided less drag forces than the model with the hole. The greater differences were reported for higher speeds, but some results were almost equal for pitch angles between $-3^{\circ}$ to $15^{\circ}$. In this case, it was not necessary to make different regressions for climbing and descending angles (see Table IV). 


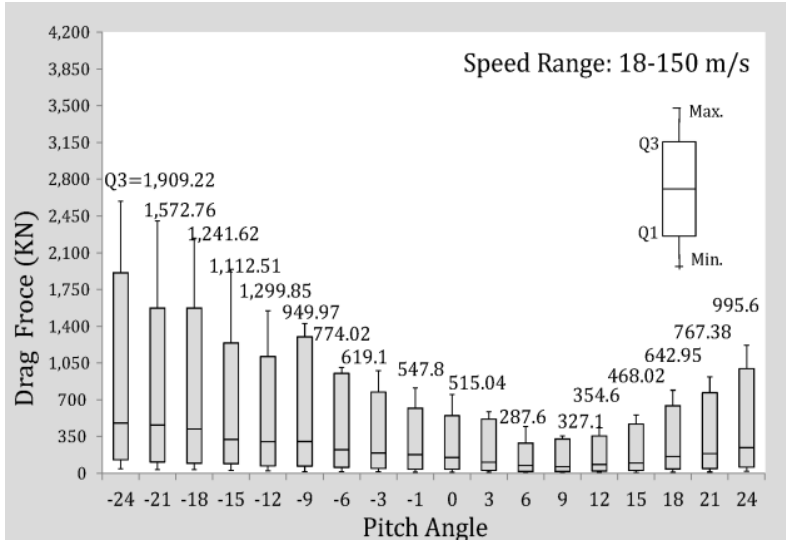

a) Model "With a Hole"

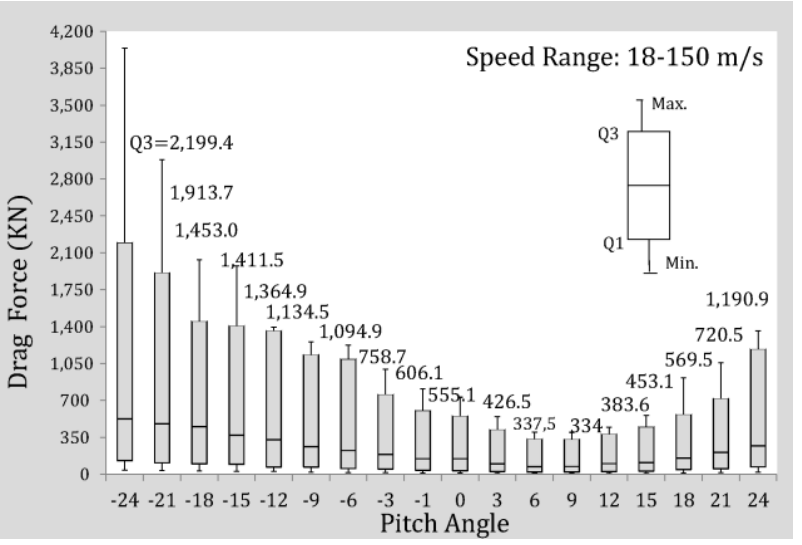

b) Model "Without a Hole"

Figure 5: Autodesk 3D Flow results for drag force in $\mathrm{kN}$ at a speed range of $18-150 \mathrm{~m} / \mathrm{s}$.
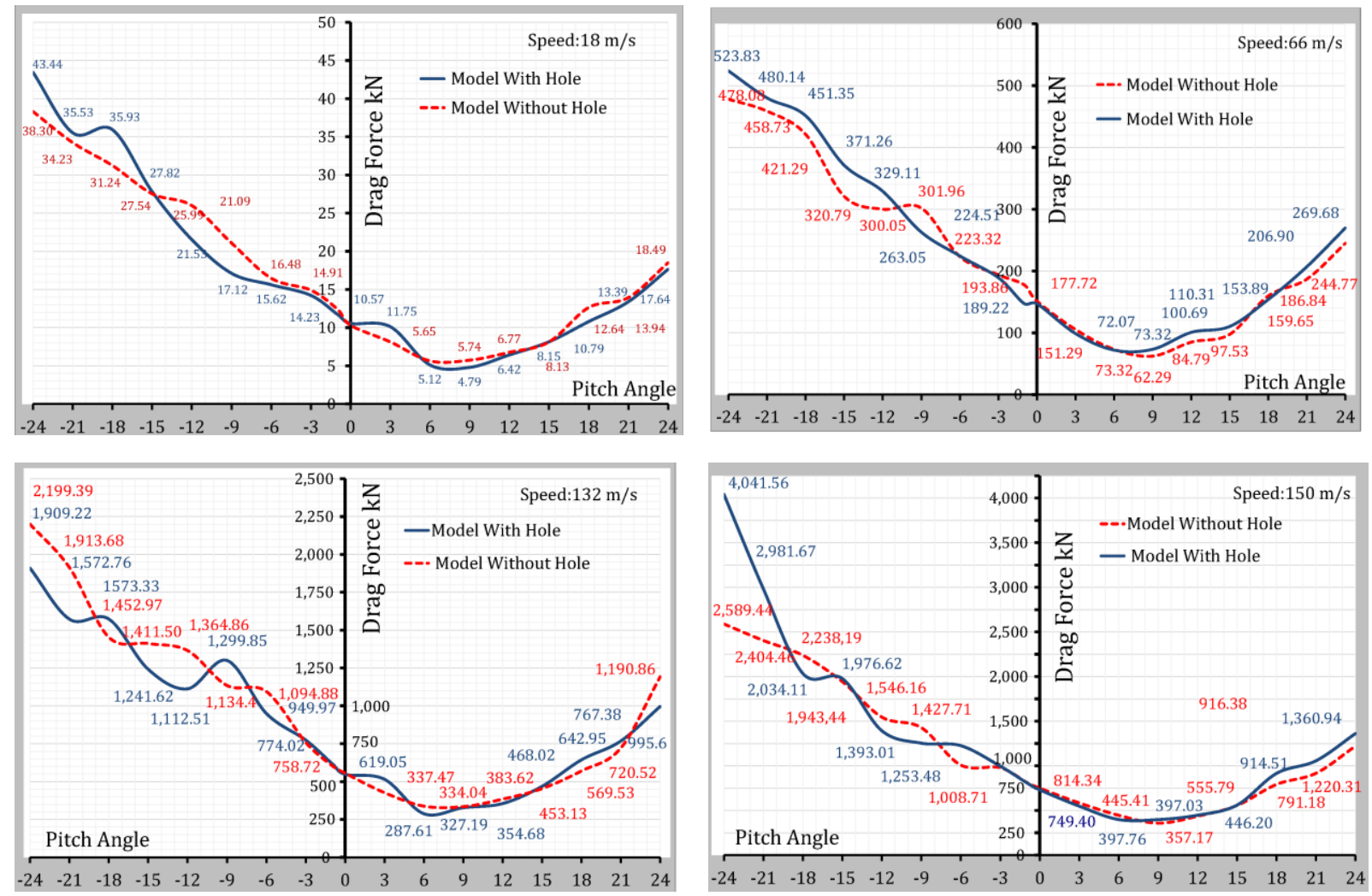

Figure 6: Examples of Autodesk 3D Flow results for drag force in $\mathrm{kN}$.

Table IV: Quadratic regressions and final results of drag force dependent on pitch angle.

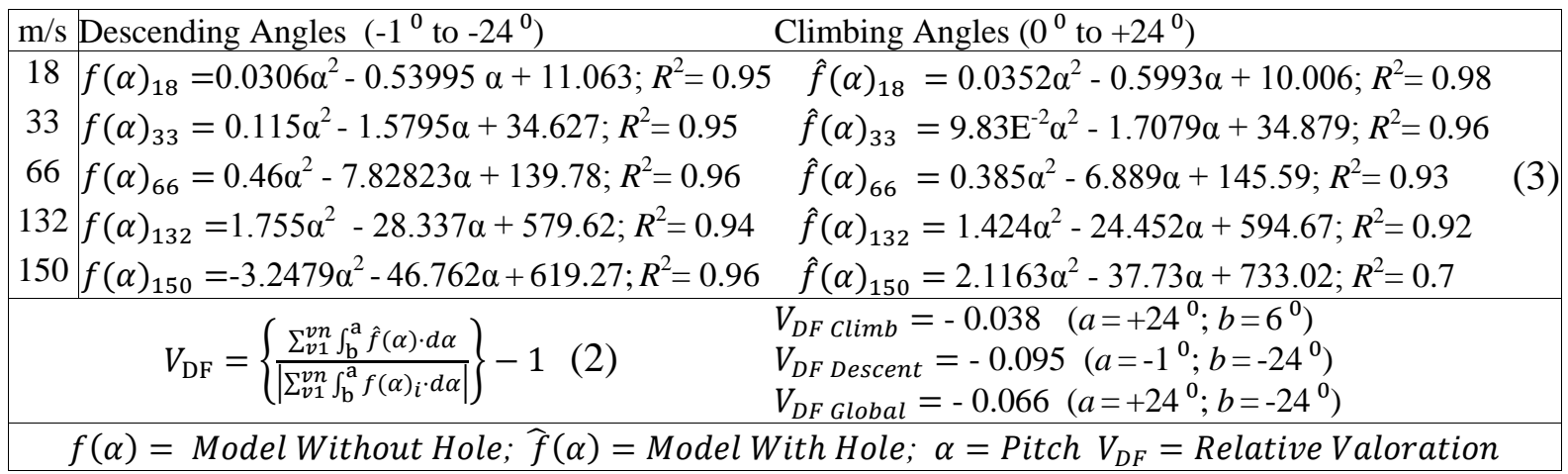




\subsection{Virtual 3D Wind Tunnel results by MicroCFD}

The simulations were set up for ascending pitch angles $\left(6^{\circ}, 10^{\circ}, 18^{\circ}\right.$ and $\left.24^{\circ}\right)$ and for a speed range between 0.2 - 0.7 Mach $(0.2,0.3,0.46$ and 0.7 Mach) in order to contrast both designs to calculate the lift force. The results were collected in Table II and plotted in a Box and Whisker chart (see Fig. 7) to provide a quick view over the lift forces obtained after almost four hours of simulation for each pitch and speed configuration setup (see graphical example of the results in Fig. 8).

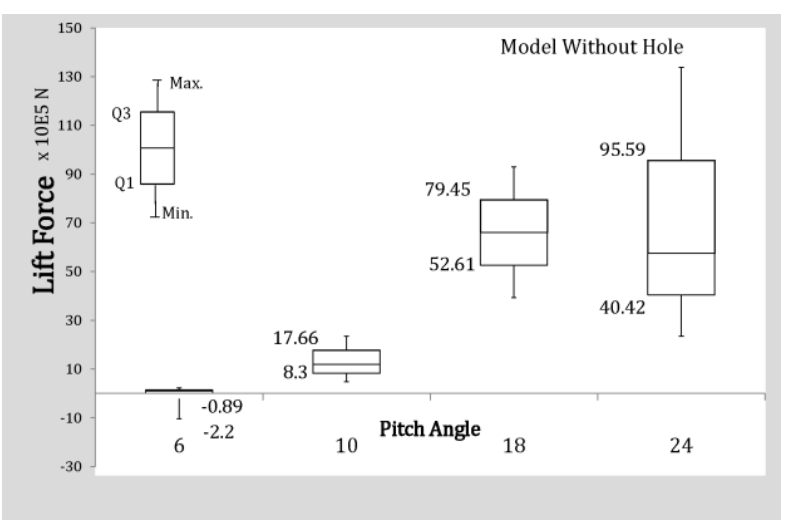

a) Model "Without a Hole"

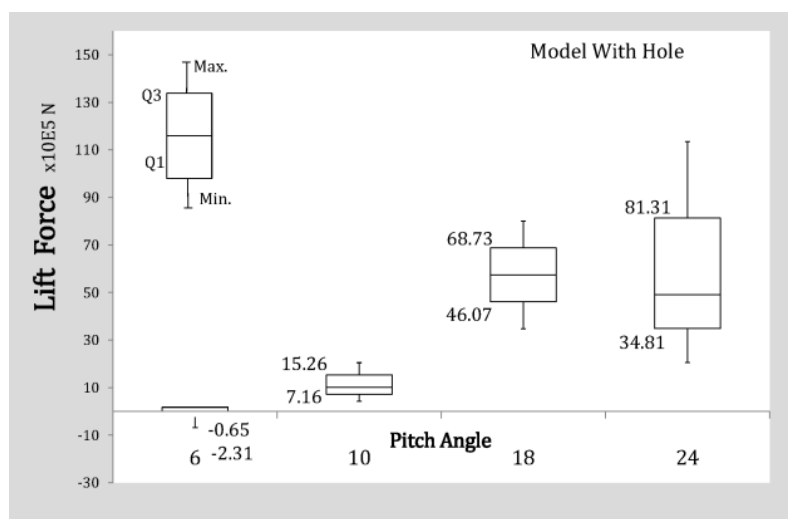

b) Model "With a Hole"

Figure 7: MicroCFD results of lift force at speed range from 0.2 to 0.7 Mach.
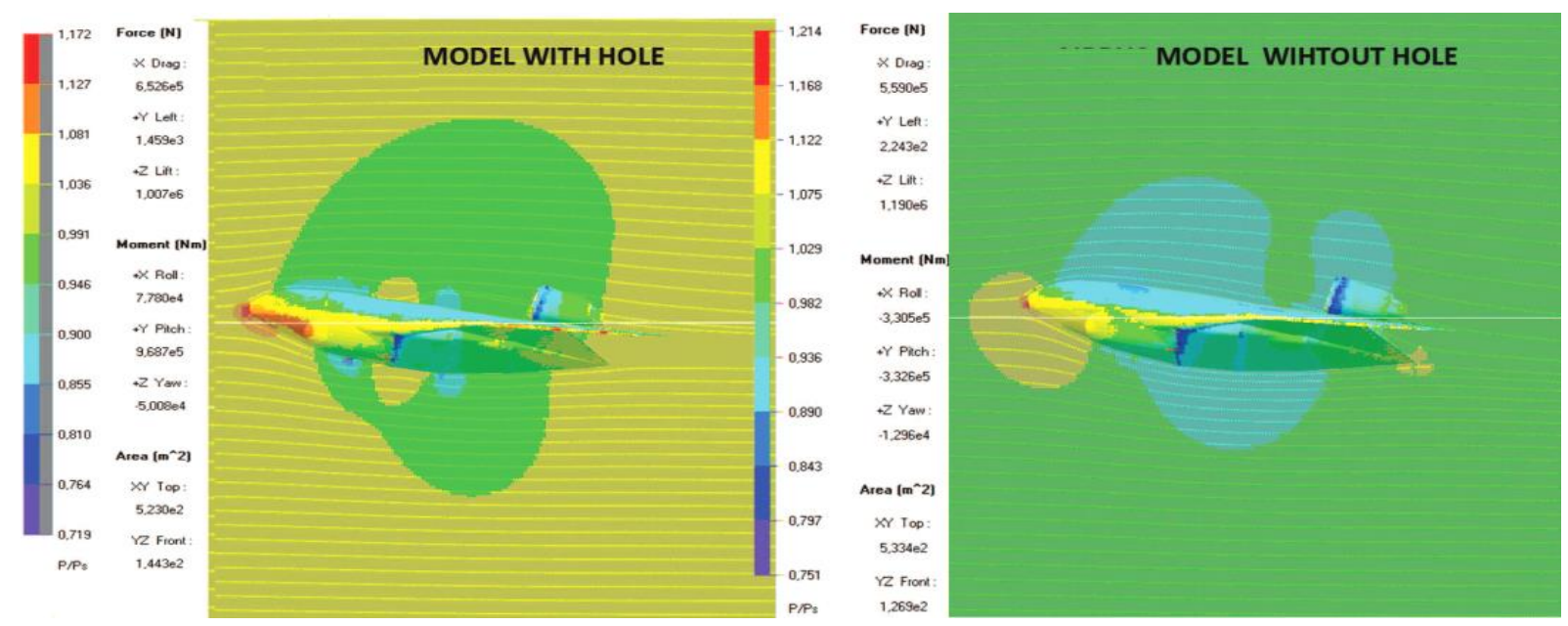

Figure 8. MicroCFD graphical results plotted at pitch angle $10^{\circ}$ and speed 0.46 Mach.

The analysis of the lift force showed that the hole addition to the design does not provide a lift improvement for all simulated cases. Once again, these simulations were not performed to define absolute values of lift force, mainly because there are several details that were not described in the patent, such as winglets, flaps, spoilers and slats that may affect the results in such a way that the negative values of lift obtained could even be positive. However, a comparison in terms of the relative variation of the lift force provided a valid estimation of the effects caused by the hole design concept when defining a global indicator of improvement, which required to adjust quadratic regressions that could be contrasted to obtain a global percentage of increasing or decreasing lift force values for the model with the hole in reference to the model without the hole (see Table V).

The result analysis indicated a negative effect because the lift force in the model with the hole decreased $18.7 \%$ for the pitch angles and speeds range selected. Notwithstanding, for a pitch angle of $6^{\circ}$ the lift force was increased by about $47 \%$, which shows a clear 
improvement for a standard flying operation. Back to the design review, it could be accepted that the hole may improve the main flying aspect of the aircraft but just for a specific range of flying parameters, which would mean that a new design half way between both models analysed could be the key to propose a design improvement. Perhaps, a model with a variable cross section hole could bring best results for any pitch and speed setup.

Table V: Quadratic regressions and final results of lift force dependent of speed flow.

\begin{tabular}{|c|c|c|}
\hline Pitch & Model Without a Hole & Model With a Hole \\
\hline 6 & $f(v)=-3 \mathrm{E}^{+6} \cdot v^{2}+57064 \cdot v ; R^{2}=0.96$ & $\hat{f}(v)=-2 \mathrm{E}^{+6} \cdot v^{2}+494307 v ; R^{2}=0.94$ \\
\hline 10 & $f(v)=4 \mathrm{E}^{+6} \cdot v^{2}+609316 \cdot v ; R^{2}=0.99$ & $\hat{f}(v)=3 \mathrm{E}^{+6} \cdot v^{2}+49867 v ; R^{2}=0.99$ \\
\hline 18 & $f(v)=1 \mathrm{E}^{+7} v^{2}+4 \mathrm{E}^{+6} v+1.38 \mathrm{E}^{+5} ; R^{2}=0.97$ & $\hat{f}(v)=1 \mathrm{E}^{+7} v^{2}+4 \mathrm{E}^{+6} v+1.17 \mathrm{E}^{+5} ; R^{2}=0.98$ \\
\hline 24 & $f(v)=3 \mathrm{E}^{+7} v^{2}-47067 v ; R^{2}=0.99$ & $\hat{f}(v)=2 \mathrm{E}^{+7} v^{2}-89367 v ; R^{2}=0.99$ \\
\hline & $V_{L F ~ G l o b a l}=\left\{\frac{\sum_{p 1}^{p n} \int_{0}^{0.7} \hat{f}(v) \cdot d v}{\left|\sum_{p 1}^{p n} \int_{0}^{0.7} f(v)_{i} \cdot d v\right|}\right\}-1$ & $\begin{array}{l}V_{\mathrm{LF} \mathrm{Global}}=-0.187 \\
V_{\mathrm{LF} P=6^{\circ}}=+0.471\end{array}$ \\
\hline
\end{tabular}

\section{CONCLUSIONS}

The design of aircraft configurations (including safety aspects) needs to be accomplished taking into account several parameters and standards related to the aircraft structure, shape and size such as wing dimensions, flapping windows, service facilities, among other complex matters. This helps understanding why final design layouts must be developed by multidisciplinary teams who work together under an innovative design context, so they may take advantage of multidisciplinary skills [16] by transferring knowledge processes when applying design concepts from one industrial environment to another.

Regarding the design of possible wing configurations for passenger planes and commercial purposes, even though it has been a study matter of high interest for the last twenty years [17], implementation processes and design aspects still need to be developed or improved. This leads aircraft manufacturers (i.e Airbus, Boeing, among others) to keep producing several new patents to take advantage of wing designs through new developments.

The drag coefficient, drag forces and lift forces may be considered crucial design indicators [18] to evaluate whatever conceptual modifications may be regarded as an improvement to be deeply analysed or not. Under this point of view, numerical simulations play an important role to test design modifications that can be professionally performed by design engineers both novice and senior with a high interest in the field of aeronautical design.

In relationship with computer simulations, the MicroCFD software offers a suitable option given the high accuracy of its results, its friendly interface and its low license cost, while the Autodesk flow design may be limited on some computational calculation aspects and presents itself as a less professional tool. However, both generate enough information to make design decisions by contrasting results in relative terms of improvement by a percentage that represents increasing or decreasing values.

In the case study analysed, it could be stated considering the Autodesk flow design that the design modification of the hole provided a drag reduction for descending angles of about $14.65 \%$ and for climbing angles of about $5.8 \%$, see Table III, Eqs. (1) and (2). In addition to these results, there was also a global reduction of the drag force for the full spectre analysed (pitch angle: -24 to $24^{\circ}$, speed: $18-150 \mathrm{~m} / \mathrm{s}$ ) of about $6.6 \%$, see Table IV, Eqs. (3) and (2). $9.5 \%$ if it was evaluated just for descending pitch $\left(-1^{0}\right.$ to $\left.-24^{0}\right)$ and $3.7 \%$ if it was evaluated just for climbing angles $\left(0^{0}\right.$ to $\left.+24^{0}\right)$. Meanwhile, MicroCFD allowed to determine that the lift force depending on flow speed was affected by a global reduction of $18.7 \%$ for the model with the hole design, see Table V, Eqs. (4) and (5); however, for a pitch angle of $6^{\circ}$, it 
produced a lift force increase of $47.06 \%$, which reveals that the hole design is a clear advantage for the standard flying pitch angle.

This case study may also open several future research lines, for example, the design overlap with other aircraft manufacturers such as Boeing through patent US\# 208/0230654 (see Fig. 9), the possible implications of noise reduction due to the hole design, passengers and cargo accommodations, serrated winglets implementation and managerial implications of the design such as new landing procedures [19]. Finally, within an academic purpose, it will be expected to design more case studies by analysing the results of implementing immersive lessons in aeronautical design, increasing their difficulty step by step under similar inverse engineering processes.

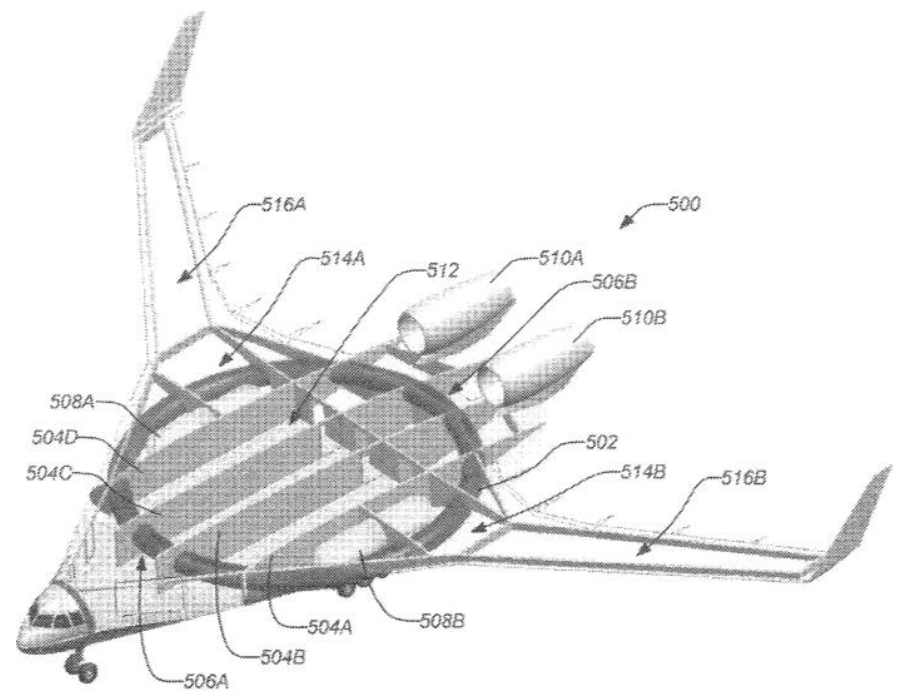

Figure 9: Boeing flying wing view. Extracted from patent \#US2008/0230654.

\subsection{Design improvement proposal}

Multidisciplinary design optimization (MDO) offers the opportunity to bring performance analysis to early stages of design by providing an expanded set of design alternatives that can be easily generated, evaluated and ranked according to multiple performance criteria by a simultaneously quantified trade-off analysis [20]. The ultimate goal of design engineering for product/process study is to enhance the performance after a redesign process to effectively improve its future use [21]. For this reason, it is required to match knowledge inside a multidisciplinary team with functional design aspects other than the minimization of processes cost [22]. As an example, when applying these concepts to this case study it was detected that the seating layout in the patent showed 122 seats placed in a radial arrangement, which seems to take advantage of the circular shape at the same time that increases the number of passenger places.

However, this layout has some disadvantages, such as some possible passenger discomfort due to side forces since some seats have to face a crosswise layout in relation to the direction of travel. The radial seats arrangement could be also a reason to complain for some passengers, not only due to travel sickness caused by side forces, but for not having enough free space at the front of the seat when seats are assigned near the centre of the aircraft.

In a multidisciplinary team, safety designers could have done an important contribution to improve the patented design just by facing all seats in the direction of aircraft travel, which in addition to offering the same leg room for all passengers, the potential number of emergency exits could have also been increased in spite of decreasing the carrying capacity by ten units (see Fig. 10). The simulations also helped to understand for some flying operations, such as take-off, how a variable cross section for the hole design (see Fig. 11) may increase the lift 
forces when closed. This fact could finally encourage the patent designers to take into consideration a new design option (one with a variable opening) to increase the range of legal coverage that is offered when patenting.

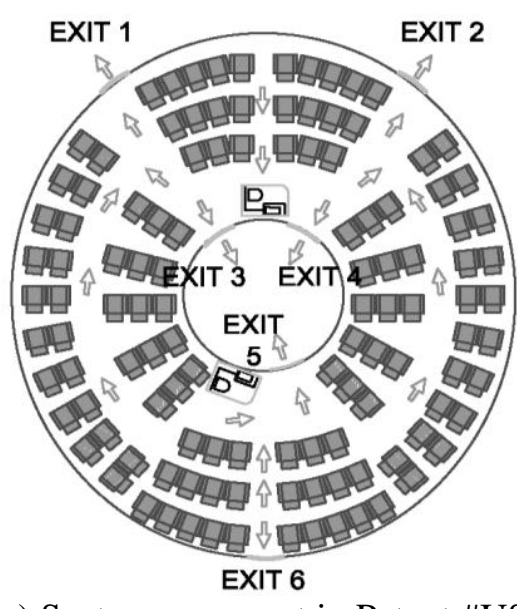

a) Seat arrangement in Patent \#US2014/0319274A1.

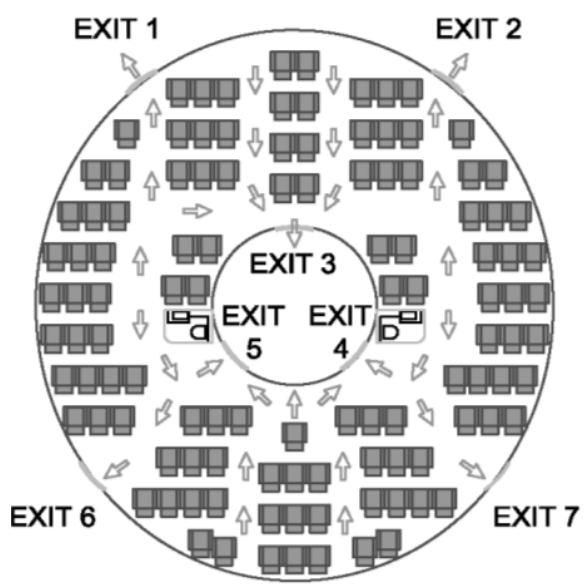

b) Seat arrangement proposal.

Figure 10: Seat arrangement proposal and exits location.
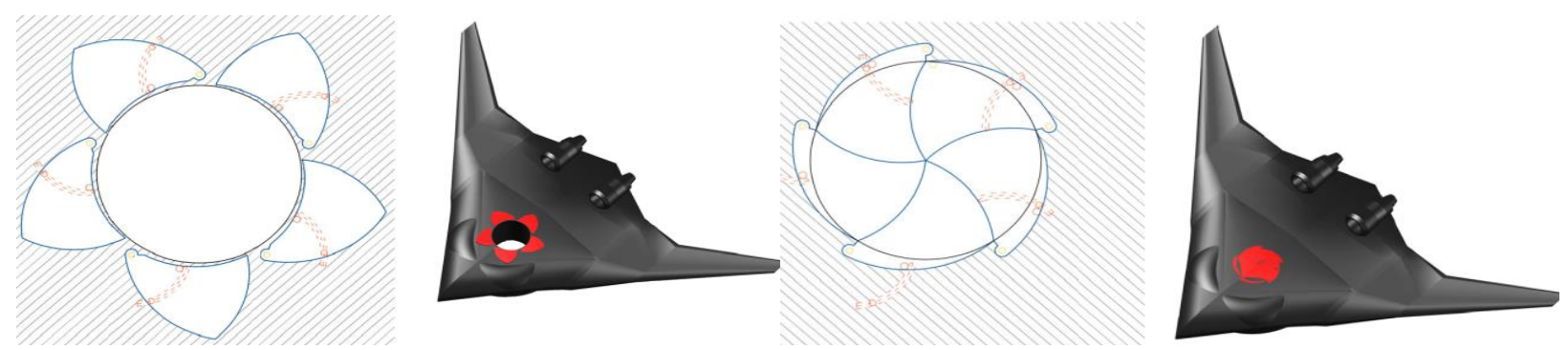

Figure 11: Iris aperture modification proposal for future research.

\section{ACKNOWLEDGEMENT}

The authors would like to express their sincere gratitude to Dr. Axel Rohde, founder and owner of MicroCFD who led this collaboration research since its first stage. This work could not have been possible to be completed without the use of MicroCFD 3D Virtual Wind Tunnel software and the support service of Dr. Rohde. More information on several Computational Fluid Dynamics Software and consulting from MicroCFD can be found at http://Microcfd.com/index.htm.

\section{REFERENCES}

[1] Safin, S.; Dorta, T.; Pierini, D.; Kinayoglu, G.; Lesage, A. (2016). Design Flow 2.0, assessing experience during ideation with increased granularity: A proposed method, Design Studies, Vol. 47, 23-46, doi:10.1016/j.destud.2016.08.002

[2] Dumas, D.; Schmidt, L. C.; Alexander, P. A. (2016). Predicting creative problem solving in engineering design, Thinking Skills and Creativity, Vol. 21, 50-66, doi:10.1016/j.tsc.2016.05.002

[3] Behm, M.; Culvenor, J.; Dixon, G. (2014). Development of safe design thinking among engineering students, Safety Science, Vol. 63, 1-7, doi:10.1016/j.ssci.2013.10.018

[4] Mohamed Ali, J. S.; Ali Omar, A.; Ali, M. A. B.; Bin Mohd Baseair, A. R. (2017). Numerical investigation of aerodynamic characteristics of high speed train, IOP Conference Series: Materials Science and Engineering, Vol. 184, Paper 012015, 1-5, doi:10.1088/1757$\underline{899 X / 184 / 1 / 012015}$

[5] Melles, G.; Howard, Z.; Thompson-Whiteside, S. (2012). Teaching design thinking: expanding horizons in design education, Procedia - Social and Behavioral Sciences, Vol. 31, 162-166, doi:10.1016/j.sbspro.2011.12.035 
[6] Starkey, E.; Toh, C. A.; Miller, S. R. (2016). Abandoning creativity: the evolution of creative ideas in engineering design course projects, Design Studies, Vol. 47, 47-72, doi:10.1016/j.destud.2016.08.003

[7] Zhang, Q.; Deniaud, I.; Lerch, C.; Baron, C.; Caillaud, E. (2016). Process modeling of innovative design using systems engineering, International Federation of Automatic Control Conference IFAC-PapersOnLine, Vol. 49, No. 12, 1579-1584, doi:10.1016/j.ifacol.2016.07.805

[8] McAlpine, H.; Cash, P.; Hicks, B. (2017). The role of logbooks as mediators of engineering design work, Design Studies, Vol. 48, 1-29, doi:10.1016/j.destud.2016.10.003

[9] Qin, N.; Vavalle, A.; Le Moigne, A.; Laban, M., Hackett, K.; Weinerfelt, P. (2004). Aerodynamic considerations of blended wing body aircraft, Progress in Aerospace Sciences, Vol. 40, No. 6, 321-343, doi:10.1016/j.paerosci.2004.08.001

[10] Bourgeois-Bougrine, S.; Buisine, S.; Vandendriessche, C.; Glaveanu, V.; Lubart, T. (2017). Engineering students' use of creativity and development tools in conceptual product design: what, when and how?, Thinking Skills and Creativity, Vol. 24, 104-117, doi:10.1016/ j.tsc.2017.02.016

[11] Law, A. (2016). The role of modelling in structural fire engineering design, Fire Safety Journal, Vol. 80, 89-94, doi:10.1016/j.firesaf.2015.11.013

[12] Cochran, D. S.; Jafri, M. U.; Chu, A. K.; Bi, Z. (2016). Incorporating design improvement with effective evaluation using the manufacturing system design decomposition (MSDD), Journal of Industrial Information Integration, Vol. 2, 65-74, doi:10.1016/j.jii.2016.04.005

[13] Holzer, D. (2016). Design exploration supported by digital tool ecologies, Automation in Construction, Vol. 72, Part 1, 3-8, doi:10.1016/j.autcon.2016.07.003

[14] Wu, D.; Rosen, D. W.; Wang, L.; Schaefer, D. (2015). Cloud-based design and manufacturing: a new paradigm in digital manufacturing and design innovation, Computer-Aided Design, Vol. 59, 1-14, doi:10.1016/j.cad.2014.07.006

[15] Love, P. E. D.; Zhou, J.; Matthews, J.; Luo, H. (2016). Systems information modelling: enabling digital asset management, Advances in Engineering Software, Vol. 102, 155-165, doi:10.1016/ j.advengsoft.2016.10.007

[16] Acharya, K.; Ghoshal, D. (2016). Nature inspired prototype design of collision avoidance aircraft system and design of a pair of wing flaps in Autodesk Maya software, Procedia Computer Science, Vol. 89, 684-689, doi:10.1016/j.procs.2016.06.036

[17] Bolsunovsky, A. L.; Buzoverya, N. P.; Gurevich, B. I.; Denisov, V. E.; Dunaevsky, A. I.; Shkadov, L. M.; Sonin, O. V.; Udzhuhu, A. J.; Zhurihin, J. P. (2001). Flying wing - problems and decisions, Aircraft Design, Vol. 4, No. 4, 193-219, doi:10.1016/S1369-8869(01)00005-2

[18] Han, Y.; Chen, H.; Cai, C. S.; Xu, G.; Shen, L.; Hu, P. (2016). Numerical analysis on the difference of drag force coefficients of bridge deck sections between the global force and pressure distribution methods, Journal of Wind Engineering and Industrial Aerodynamics, Vol. 159, 65-79, doi:10.1016/j.jweia.2016.10.004

[19] Filippone, A. (2017). Options for aircraft noise reduction on arrival and landing, Aerospace Science and Technology, Vol. 60, 31-38, doi:10.1016/j.ast.2016.10.027

[20] Eve Lin, S.-H.; Gerber, D. J. (2014). Designing-in performance: a framework for evolutionary energy performance feedback in early stage design, Automation in Construction, Vol. 38, 59-73, doi:10.1016/j.autcon.2013.10.007

[21] Ullah, I.; Tang, D.; Yin, L. (2016). Engineering product and process design changes: a literature overview, Procedia CIRP, Vol. 56, 25-33, doi:10.1016/j.procir.2016.10.010

[22] Yao, X.; Ki Moon, S.; Bi, G. (2017). Multidisciplinary design optimization to identify additive manufacturing resources in customized product development, Journal of Computational Design and Engineering, Vol. 4, No. 2, 131-142, doi:10.1016/j.jcde.2016.10.001 